eCommons@AKU

Department of Paediatrics and Child Health

Division of Woman and Child Health

January 2018

\title{
Partnerships for child health: capitalising on links between the sustainable development goals
}

Yulia Blomstedt

Umeå University,Sweden

Zulfiqar Ahmed Bhutta

Aga Khan University, zulfiqar.bhutta@aku.edu

Johan Dahlstrand

Global Health Law Georgetown University Law Center,USA

Peter Friberg

Global Health Law Georgetown University Law Center,USA

Lawrence O. Gostin

Global Health Law Georgetown University Law Center,USA

See next page for additional authors

Follow this and additional works at: https://ecommons.aku.edu/

pakistan_fhs_mc_women_childhealth_paediatr

Part of the Pediatrics Commons

\section{Recommended Citation}

Blomstedt, Y., Bhutta, Z. A., Dahlstrand, J., Friberg, P., Gostin, L. O., Nilsson, M., Sewankambo, N. K., Tomson, G., Alfvén, T. (2018).

Partnerships for child health: capitalising on links between the sustainable development goals. BMJ:British medical journal, 360 .

Available at: https://ecommons.aku.edu/pakistan_fhs_mc_women_childhealth_paediatr/309 
Authors

Yulia Blomstedt, Zulfiqar Ahmed Bhutta, Johan Dahlstrand, Peter Friberg, Lawrence O. Gostin, Måns Nilsson, Nelson K. Sewankambo, Göran Tomson, and Tobias Alfvén 


\title{
Partnerships for child health: capitalising on links between the sustainable development goals
}

Yulia Blomstedt and colleagues evaluate the opportunities to improve child health through cross sector collaboration

\author{
Yulia Blomstedt researcher ${ }^{1}$, Zulfiqar A Bhutta professor ${ }^{2}{ }^{3}$, Johan Dahlstrand researcher ${ }^{4}$, Peter \\ Friberg professor ${ }^{45}$, Lawrence O Gostin professor ${ }^{6}$, Måns Nilsson professor ${ }^{7}{ }^{8}$, Nelson $\mathrm{K}$ \\ Sewankambo professor ${ }^{9}$, Göran Tomson professor ${ }^{4} 1{ }^{11}$, Tobias Alfvén associate professor ${ }^{411} 12$
}

${ }^{1}$ Epidemiology and Global Health Unit, Department of Public Health and Clinical Medicine, Umeå University, Umeå, Sweden; ${ }^{2}$ Centre of Excellence in Women and Child Health, Aga Khan University, Karachi, Pakistan; ${ }^{3}$ Centre for Global Child Health, Hospital for Sick Children, Toronto, Canada; 4Swedish Institute for Global Health Transformation (SIGHT), Royal Swedish Academy of Sciences, Stockholm, Sweden5Institute of Medicine, Sahlgrenska Academy, University of Gothenburg, Sweden; ${ }^{6}$ O'Neill Institute for National and Global Health Law Georgetown University Law Center, Washington, DC, USA; ${ }^{7}$ Stockholm Environment Institute, Stockholm, Sweden; ${ }^{8} \mathrm{KTH}$ Royal Institute of Technology, Stockholm, Sweden; ${ }^{9} \mathrm{Makerere}$ University, School of Medicine, College of Health Sciences, Uganda; ${ }^{10}$ Departments of Learning, Informatics, Management, Ethics, and Public Health Sciences, Karolinska Institutet, Stockholm, Sweden; ${ }^{11}$ Department of Public Health Sciences, Karolinska Institutet, Stockholm, Sweden ; ${ }^{12}$ Sachs' Children and Youth Hospital, Stockholm, Sweden

In 2015 the UN General Assembly adopted the sustainable development goals (SDGs) as part of a transformative universal framework for global development: the 2030 agenda. ${ }^{1}$ Since the goals are interconnected, ${ }^{2-6}$ they have to be tackled in an integrated way. As well as ensuring that goals are reached efficiently, integration can avoid adverse effects from action to meet other targets and highlight trade-offs. ${ }^{4}$

Integrated action relies on national and international partnerships with a broad range of organisations-including national governments, local authorities, international institutions, business, civil society organisations, foundations,

philanthropists, social impact investors, scientists, and citizens. ${ }^{7}$ However, it is not always clear who should partner with whom and on what grounds. Making these decisions-and developing integrated action plans, strategies, and policies-requires an understanding of the patterns of interaction between SDGs.

We use the example of child health to explore how assessment of the links between SDGs can be used to guide multisectoral partnerships. The importance of partnerships within the health sector is well established for child health, given the role of maternal health and nutrition in stillbirths, newborn health, and survival, as well as early child growth and development. By contrast, partnerships with other sectors have received much less attention. Efforts to include social determinants of health in development of health systems and public health have begun to provide crucial information. For example, the health in all policies $(\mathrm{HiAP})^{8}$ initiative assessed multisectoral public policies, their health implications, synergies, and potential adverse outcomes. The SDGs offer a new opportunity for collaboration between the health sector and the rest of society and, hopefully, the momentum to move from mostly talk to action.

\section{SDG links as lever for improved child health strategies}

The connections between child health (0-18 years old) and development and other priorities in the SDGs are strong and reciprocal. The survival, health, and wellbeing of children are essential to end extreme poverty and promote development and resilience. ${ }^{9}$ At the same time, social, economic, political, environmental, and cultural determinants have important effects on child health. ${ }^{10}{ }^{11}$ Consequently, interventions beyond the health sector will accelerate progress on child health ${ }^{10}$ while investments in child health are crucial to reach multiple SDGs. However, this interdependence is not reflected in the formulation of SDG targets and indicators.

Although many SDGs have overlapping areas of concern, few of the goals directly integrate other sectors and reflect all three dimensions of development (social, economic, and environmental) either in the goals themselves or in the targets. This lack of integration risks reinforcing the current silo approach to development. ${ }^{12}$ For example, all too often people with a medical or public health background do not reach beyond the health sector to find effective and efficient solutions to health challenges. This was a problem in the implementation of the millennium development goals. ${ }^{13}$ 
Highlighting the interdependence between the health goal (SDG3) and targets in the other SDGs has the potential to transform the approach to child health. Once we have identified the connections we need to create bridges between them and develop and facilitate multisectoral interventions.

Although the 2030 agenda is not legally binding, virtually all UN member states have agreed to implement it. ${ }^{1}$ Concrete guidance on linking child health with other SDGs in the agenda would provide a lever to push governments to take multisectoral action in this and other areas. It would also provide a substantive basis for creating multistakeholder partnerships on child health.

\section{Identifying suitable partners in other sectors}

Sectors outside healthcare that are of crucial importance for child health include education, finance and social protection, labour and trade, agriculture, and transport. ${ }^{9}$ The first step to ensure multisectoral action is to identify key interventions in these non-health sectors that contribute to health outcomes. ${ }^{10}$ Besides SDG3 on health, 24 targets specifically mention children (Boxed Text on page 3box 1) and have the potential to improve child health. 
Box 1Sustainable development goals (besides SDG3) that mention children

\section{SDG 1: No poverty}

1.2 By 2030 , reduce at least by half the proportion of men, women, and children of all ages living in poverty in all its dimensions according to national definitions

1.3 Implement nationally appropriate social protection systems and measures for all, including floors, and by 2030 achieve substantial coverage of the poor and the vulnerable

Indicator 1.3.1 Proportion of population covered by social protection floors/systems, by sex, distinguishing children, unemployed people, older people, people with disabilities, pregnant women, newborns, work injury victims, poor people, and vulnerable groups

\section{SDG 2: Zero hunger}

2.1 By 2030, end hunger and ensure access by all people, in particular the poor and people in vulnerable situations, including infants, to safe, nutritious, and sufficient food all year round

2.2 By 2030 , end all forms of malnutrition, including achieving, by 2025 , the internationally agreed targets on stunting and wasting in children under 5 years of age, and address the nutritional needs of adolescent girls, pregnant and lactating women and older people

\section{SDG 4: Quality education}

4.1 By 2030, ensure that all girls and boys complete free, equitable, and quality primary and secondary education leading to relevant and effective learning outcomes

4.2 By 2030 , ensure that all girls and boys have access to quality early childhood development, care, and pre-primary education so that they are ready for primary education

4.4 By 2030 , substantially increase the number of youth and adults who have relevant skills, including technical and vocational skills, for employment, decent jobs, and entrepreneurship

4.5 By 2030 , eliminate gender disparities in education and ensure equal access to all levels of education and vocational training for the vulnerable, including people with disabilities, indigenous peoples, and children in vulnerable situations

4.6 By 2030 , ensure that all youth and a substantial proportion of adults, both men and women, achieve literacy and numeracy

4a Build and upgrade education facilities that are child, disability, and gender sensitive and provide safe, non-violent, inclusive, and effective learning environments for all

\section{SDG 5: Gender equality}

5.1 End all forms of discrimination against all women and girls everywhere

5.2 Eliminate all forms of violence against al women and girls in the public and private spheres, including trafficking and sexual and other types of exploitation

5.3 Eliminate all harmful practices, such as child, early and forced marriage, and female genital mutilation

5c Adopt and strengthen sound policies and enforceable legislation for the promotion of gender equality and the empowerment of all women and girls at all levels

\section{SDG 6: Clean water and sanitation}

6.2 By 2030 , achieve access to adequate and equitable sanitation and hygiene for all and end open defecation, paying special attention to the needs of women and girls and those in vulnerable situations

SDG 8: Decent work and economic growth
8.5 By 2030 , achieve full and productive employment and decent work for all women and men, including for young people and people with disabilities, and equal pay for work of equal value

8.6 By 2020 , substantially reduce the proportion of youth not in employment, education, or training

8.7 Take immediate and effective measures to eradicate forced labour, end modern slavery and human trafficking, and secure the prohibition and elimination of the worst forms of child labour, including recruitment and use of child soldiers, and by 2025 end child labour in all its forms 8 b By 2020, develop and operationalise a global strategy for youth employment and implement the global jobs pact of the International Labour Organisation

\section{SDG 11: Sustainable cities and \\ communities}

11.2 By 2030, provide access to safe, affordable, accessible, and sustainable transport systems for all, improving road safety, notably by expanding public transport, with special attention to the needs of those in vulnerable situations, women, children, people with disabilities, and older people

11.7 By 2030, provide universal access to safe inclusive, and accessible green and public spaces, in particular for women and children, older people and people with disabilities

\section{SDG 13: Climate action}

13b Promote mechanisms for raising capacity for effective climate change-related planning and management in least developed countries and small island developing States, including focusing on women, youth, and local and marginalized communities

\section{SDG 16: Peace, justice, and strong institutions}

16.2 End abuse, exploitation, trafficking, and all forms of violence against and torture of children 16.9 By 2030, provide legal identity for all, including birth registration

Indicator 16.9.1 Proportion of children under 5 years of age whose births have been registered with a civil authority, by age

However, the health sector should go beyond being a passive recipient and monitor of health effects generated by action on other SDGs. Health is not merely an outcome but also a driver of change in other sectors and has the potential to help achieve multiple SDGs $(\Downarrow)$. For example, improved child health removes resource constraints for households and communities (SDG 1), such that parents can spend time on employment (SDG 8) and spend money on non-health needs (eg, SDGs 2 and 4). Actors in sectors traditionally responsible for these SDGs would directly benefit from collaborating with the actors in child health. Boxed Text on page 3Box 2 gives an example of such synergetic collaboration.

Box 2Synergistic effects of multisectoral action: cas study of conditional cash transfer strategies Conditional cash transfer (CCT) programmes provide a cash payment to poor families or individuals in exchange for them taking actions intended to improve their wellbeing. The conditions, evidence based interventions, may include such things as consistent school attendance, prenatal health check-ups, and vaccinations for children. Hence, the programmes have potential to diminish poverty (SDG1) and improve the health outcomes (SDG3) and socioeconomic status of children and their families (SDGs 4, 5, 8).

The first larger scale CCT programmes was PROGRESA in Mexico. It began in 1997, partly in 


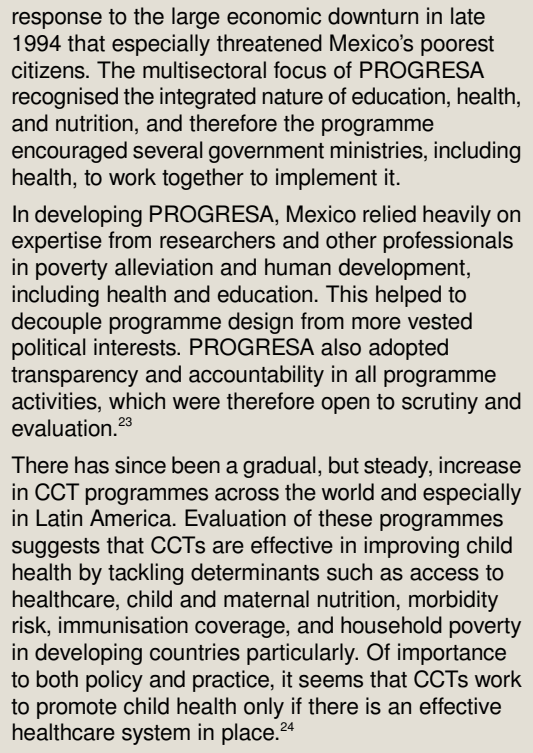

\section{Finding common grounds for multisectoral partnerships}

Another approach to establishing multisectoral partnerships is through understanding the patterns of interaction between SDGs. This could be achieved through the following steps:

Mapping interactions between targets - Links between, in this case, child health and targets in other SDGs should be guided by scientifically shown associations between child health and other factors. Such mappings reveal potential partners and areas of overlapping responsibility or interest. Child health targets include SDG 3.1 (maternal mortality), 3.2 (newborn and child mortality), 3.7 (universal access to sexual and reproductive health-care services).

Assessing the direction of the identified interaction is important for establishing the grounds for collaboration. Is child health an outcome or a prerequisite of achieving another target, or is the link reciprocal? Establishing collaboration on targets where the link is reciprocal would have synergetic effects, propelling the achievement of both child health and the linked target.

Assessing the strength of associationor identified interaction is important for prioritisation, identifying where multisectoral collaboration is crucial for achieving the target(s). As with any implementation, it is important to clarify the main groups involved as well as their power relations.

The SDG interactions framework developed by Nilsson and colleagues supports such explorative processes and suggests scoring connections on a seven point scale (Boxed Text on page 4box 3$).{ }^{14}$ The position and nature of the interaction may vary depending on the context within which the interaction occurs.

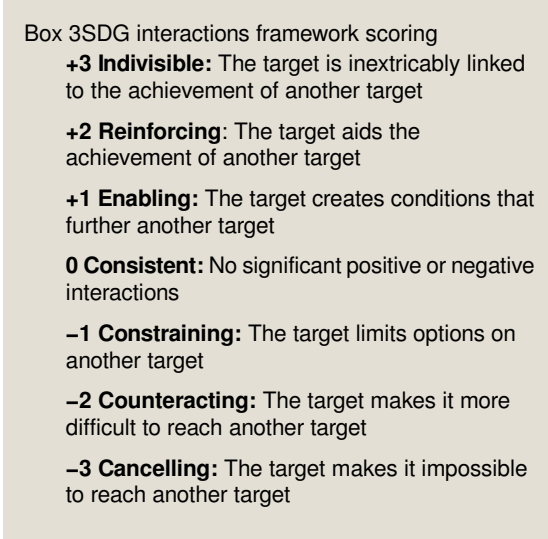

We used the framework to assess interactions with child health in other SDGs, informed by analysis of relevant sources. ${ }^{4-10}$ Between us we have expertise in environmental science, law, medicine, paediatrics, health systems research, development economics, and global health. Each author assessed the linkage with the identified targets, and a final score was agreed by discussion.

Our generic (not context specific) analysis suggests that collaboration with those implementing SDGs 1 (no poverty), 2 (zero hunger), 4 (quality education), 5 (gender equality), 8 (decent work and economic growth), and 17 (partnerships for the goals) could be cost effective because of the reciprocal link with child health ( $\Downarrow$ and supplement 2 on bmj.com) The last column of $\Downarrow$ gives examples of such synergistic multisectoral action, taken from the Every Woman Every Child report. ${ }^{9}$

The analysis also suggested that multisectoral collaboration on the following targets is essential for sustainable progress on child health (score +3 ): 1.1 (eradicate extreme poverty), $\bullet 1.2$ (reduce at least by half the proportion of men, women, and children of all ages living in poverty in all its dimensions according to national definitions), 2.1 (end hunger), 2.2 (end all forms of malnutrition), 4.1 (ensure that all girls and boys complete free, equitable, and quality primary and secondary education), 6.1 (achieve universal and equitable access to safe and affordable drinking water), 6.2 (achieve access to adequate and equitable sanitation and hygiene), and 11.1 (ensure access for all to adequate, safe, and affordable housing and basic services and upgrade slums).

An example where multisectoral collaboration has large potential is the water, sanitation, and hygiene (WASH) programmes (SDGs 6.1 and 6.2). Despite the large health benefits, these programmes are still often viewed as an infrastructure led issues, the responsibility for which lies outside health system. This perception is reinforced by the often curative focus of the health sector and acts as a barrier to integration of WASH aspects in health strategies and programmes (Boxed Text on page 5box 4). Another difficulty is that the benefits of WASH programmes may accrue over time and so investment may not provide short term health and nutrition benefits. More examples of successful multisectoral interventions on determinants of reproductive, maternal, newborn, child, and adolescent health can be found in the SDG knowledge platform (https://sustainabledevelopment. un.org/sdinaction). 


\begin{abstract}
Box 4Water, sanitation and hygiene-how multisectoral action can improve child health Creating multisectoral interventions that address water, sanitation, and hygiene (WASH) and health, and integrating WASH into existing frameworks and agendas for health are critical for improving child health through reduced exposure to enteric infections and improved nutrition. The interventions may also improve gender equality (SDG 5) and human rights (SDG 16). Girls are disproportionally affected, missing school because of walking great distances to carry water for household use, as well as lacking adequate sanitation and hygiene facilities in schools to allow them to manage their menstruation. Inadequate WASH facilities are also associated with sexual assault and gender based violence, where toilets are unavailable or unsafe..$^{15}$

Integrating programmes to improve WASH into existing health programmes has been shown to be successful. Childhood vaccination has one of the highest coverage rates among child survival interventions. In Kenya hygiene interventions and education were integrated into the vaccination services by nurses or community health workers with good results. ${ }^{16}$ The interventions led to an improvement in hygiene indicators such as knowledge and use of household disinfection of water and had high acceptance and uptake in both rural and urban communities.

Another example of multisectoral partnerships is Laos, where WASH and ECCD (Early Childhood Care and Development) programmes worked closely together at district and village level. In this model multiple sectors worked together in planning and monitoring, with implementation largely carried out individually by each sector-sometimes referred to as "think multisectorally, act sectorally." The experience highlighted the importance of all sectors in multisectoral programmes having a shared understanding of purpose and equitable access to resources to do their work as well as having effective leadership and clear organisational arrangements in place. ${ }^{17}$
\end{abstract}

Our analysis found few negative interactions, and none scored -3 . The limited number of trade-offs with health has been noted previously ${ }^{18}$ and contrasts with domains such as infrastructure development, export promotion, and ecosystem conservation. However, connections between SDGs are highly context dependent, ${ }^{19}$ and conflicts between child health and other targets may still arise during implementation. To be relevant and transferable to any single setting, our analysis therefore needs to be repeated with sensitivity to political, economic, geographical, and social context by a multidisciplinary and multisectoral group of local stakeholders. Although the direction of the associations between child health and other targets in most contexts will remain the same, the strength and nature of the interaction may shift. ${ }^{18}$

For example, the link between ensuring access to modern energy (SDG 7.1) and child health is evident and true globally. However, whether the pursuit of modern energy is positive or negative for child health depends on context $(\Downarrow)$. In places where basic energy access is already achieved, further efforts may not affect child health. If modern energy meant exclusively renewables, the link could be viewed as positive $(+1$ or +2$)$. However, if energy access is instead pursued using polluting fossil or solid biomass fuels, they could have negative effect on child health (-2).

\section{New ways of thinking}

Our purpose here has been to introduce a way of thinking about interactions and start a debate about how such thinking can contribute to better dialogue and building partnerships. Through an integrated approach, the SDGs offer substantial opportunities to improve child health worldwide. ${ }^{20}$ The knowledge base for such action requires further development. Collaboration between the scientific community, decision makers, and implementers is vital ${ }^{21}$ to ensure that policies and action are evidence based and that child health takes its rightful place at the heart of the development agenda.

Understanding and communicating interconnections between SDGs as presented in this article can form a basis for bridging science and decision making. Various analytical and process oriented tools and methods can be used to support the identification and scoring, depending on the stakeholders involved and the exact purpose of the exercise. Academic institutions and think tanks can facilitate this process, ${ }^{22}$ providing the knowledge and evidence needed to government ministries and their implementing agencies, and supporting the exploration and communication of interactions across disciplines, sectors, and borders for transformative action on child health nationally and globally.

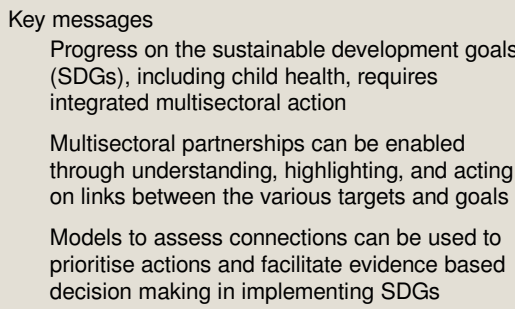

Contributors and sources: All authors have contributed to this article with their expertise in global health, including global health law and justice (LOG), women's and children's health (ZAB, TA, PF, GT), health systems (GT, NKS), development economics (JD) and SDG linkages (MN, YB). Sarah Dickin at Stockholm Environment Institute provided important input to box 4 on water, sanitation and hygiene (WASH). All authors have read and approved the final draft. YB is the guarantor. Competing interests: We have read and understood BMJ policy on declaration of interests and have no relevant interests to declare. The analysis was funded by the Bill \& Melinda Gates Foundation OPP1162011.

Provenance and peer review: Not commissioned; externally peer reviewed.

Transforming our world: the 2030 agenda for sustainable development. UN General Assembly, 2015.

2 LeBlanc D. Towards integration at last? The sustainable development goals as a network of targets. UN Department of Economic and Social Affairs, 2015.

3 WaageJYapCBellS. Governing the UN sustainable development goals: interactions, infrastructures, and institutions. Lancet Glob Health2015;3:e251-2. doi:10.1016/S2214-109X(15)70112-925833663

4 International Council of Science. Review of targets for the sustainable development goals: the science perspective. ICSU, ISSC, 2015.

5 United Nations. A nexus approach for the SDGs: interlinkages between the goals and targets: UN, 2016.

6 CutterAOsbornDRomanoJUllahF. Sustainable development goals and integration: achieving a better balance between the economic, social and environmental dimensions.Stakeholder Forum, 2015.

7 United Nations. Addis Ababa action agenda of the third international conference on financing for development, 2015. http://www.un.org/esa/ffd/wp-content/uploads/2015/08/ AAAA Outcome.pdf

8 WHO. Health in all policies: Helsinki statement. Framework for country action. 2013.http: //apps.who.int/iris/bitstream/10665/112636/1/9789241506908_eng.pdf?ua=1

9 Every Woman Every Child. Global strategy for women's, children's and adolescents' health (2016-2030). 2015. http://www.everywomaneverychild.org/global-strategy/

10 RasanathanKDamjiNAtsbehaT. Ensuring multisectoral action on the determinants of reproductive, maternal, newborn, child, and adolescent health in the post-2015 era. BMJ2015;351:h4213. doi:10.1136/bmj.h421326371220

11 Commission on Social Determinants of Health. Closing the gap in a generation: Health equity through action on the social determinants of health. Final report of the Commission on Social Determinants of Health. World Health Organization, 2008.

12 Marmot M, Allen J, Goldblatt P, et al. Fair society, healthy lives. The Marmot review. Commission on Social Determinants of Health, 2010.

13 FehlingMNelsonBDVenkatapuramS. Limitations of the millennium development goals: a literature review. Glob Public Health2013;8:1109-22. doi:10.1080/17441692.2013.84567624266508

14 NilssonMGriggsDVisbeckM. Policy: Map the interactions between sustainable development goals. Nature2016;534:320-2. doi:10.1038/534320a27306173 
15 Partnership for Maternal, Newborn and Child Health. Knowledge summary 30. Water, sanitation and hygiene-the impact on RMNCH. 2014. http://www.who.int/pmnch/ knowledge/publications/summaries/ks30/en/index1.html

16 RymanTKBriereECCartwrightE. Integration of routine vaccination and hygiene interventions: a comparison of 2 strategies in Kenya. J Infect Dis2012;205(Suppl 1):S65-76. doi:10.1093/infdis/jir77722315389

17 Plan. Multisectoral education and WASH programming in the Lao PDR. A case study of Plan's ECCD and WASH programs in Bokeo province. 2014.https://plan-international.org/ sites/files/plan/media_wysiwyg/plan-lao-nutrition-multisectoralprogramming-convergenceevaluation-eng-may14.pdf

18 NilssonM. Important interactions among the sustainable development goals under review at the high-level political forum 2017.Stockholm Environment Institute, 2017.

19 StenbergKHanssenOEdejerTT. Financing transformative health systems towards achievement of the health Sustainable Development Goals: a model for projected resource achievement of hesource needs in 67 low-income and middle-income countries. Lancet Glob Health2017;5:e875-87.
doi:10.1016/S2214-109X(17)30263-228728918
20 TaylorSWilliamsBMagnusDGoenkaAModiN. From MDG to SDG: good news for global child health?Lancet2015;386:1213-4. doi:10.1016/S0140-6736(15)00300-126460639 21 GhaffarALangloisEVRasanathanKPetersonSAdedokunLTranNT. Strengthening health systems through embedded research. Bull World Health Organ2017;95:87. doi:10.2471/BLT.16.18912628250505

22 KickbuschlHanefeldJ. Role for academic institutions and think tanks in speeding progress on sustainable development goals. BMJ2017;358:j3519. doi:10.1136/bmj.j351928739591

23 Gantner L. Case study \#5-1. PROGRESA: an integrated approach to poverty alleviation in Mexico. 2007. https://ecommons.cornell.edu/xmlui/bitstream/handle/1813/52625/dns_ in Mexico. 2007. https://ecommons.

24 Owusu-AddoECrossR. The impact of conditional cash transfers on child health in lowand middle-income countries: a systematic review. Int J Public Health2014;59:609-18. doi:10.1007/s00038-014-0570-x24898173

Published by the BMJ Publishing Group Limited. For permission to use (where not already granted under a licence) please go to http://group.bmj.com/group/rights-licensing/ permissions 


\section{Tables}

Table 1| SDG3 targets crucial for the achievement of other SDGs. Summary table based on the scientific analysis presented by the International Council for Science and International Social Science Council4

\begin{tabular}{lcl} 
SDG & Relevant SDG3 target* & \multicolumn{1}{c}{ Motivation/link } \\
SDG 1: No poverty & 3.8 & Access to free health care is fundamental to eradicating poverty \\
\hline SDG 2: Zero hunger & $3.1,3.2,3.3,3.9,3 . b, 3 . c, 3 . d$ & $\begin{array}{l}\text { Reduction of maternal, infant, and under } 5 \text { deaths and the reduction of disease } \\
\text { contributes to SDG2 }\end{array}$ \\
\hline SDG 5: Gender equality & $3.1,3.2,3.3,3.4,3.5,3.6,3.7,3.8,3 c$ & $\begin{array}{l}\text { Attention to women and children in health initiatives is vital given their central roles } \\
\text { in both social and biological reproduction and their need for access to health related } \\
\text { services }\end{array}$ \\
\hline $\begin{array}{l}\text { SDG 8: Decent work and economic } \\
\text { growth }\end{array}$ & $\begin{array}{l}\text { Expected longevity at birth can be a measure of quality of life as a main outcome } \\
\text { of sustainable and inclusive economic growth }\end{array}$ \\
\hline
\end{tabular}




\section{Table 2| Example of interactions between child health and targets under other SDGs (see supplement 2 on bmj.com for a complete list of} relevant targets)

\section{Key dimension/target of goal Score, ${ }^{*}$ description of key interaction Eradicate extreme poverty (1.1) \\ +3 , Getting out of extreme poverty is inseparable from better child health, as health problems drive people into poverty and poverty leads to-for example, reduced access to health services and increased malnutrition}

\section{Type of \\ Policies and interventions to enhance or mitigate interaction} interaction

Reciprocal

Reduce poverty, including through the use of gender and child sensitive cash transfer programmes designed to improve health

Implement social protection and assistance measures ensuring access for women, children, and adolescents

Strengthen access to health insurance and insurance related to other essential services and goods to decrease the effect of catastrophic out-of-pocket spending

Ensure access to safe, nutritious +3 , Sufficient, nutritious food is integral to Reciprocal and sufficient food by all, improved child health including infants (2.1)

+3 , Education, particularly of girls and women would be transformational in all aspects of health, productivity, and development secondary education for all girls and boys (4.1)
Ensure quality primary and

Reciprocal Integrate early child development interventions in child health services, childcare services, and preschool education

Enable girls and boys to complete quality primary and secondary education, including by removing barriers that suppress demand for education

Ensure access to education in humanitarian settings and in marginalised and hard-to-reach areas, including for people with disabilities

Eliminate all forms of violence against all women and girls

(5.2), eliminate all harmful

+2 , Achieving this SDG aids the Health is achievement of child health

outcome

Enforce legislation to prevent violence against women and girls and ensure an appropriate response when it occurs

Enact a legal framework for protection, ensuring universal access to legal services (including to register human rights violations and have recourse to remedial action against them)

Implement social protection and assistance measures ensuring access for women, children, and adolescents

Provide protection services for women, children, and adolescents that are age and sex appropriate

\begin{tabular}{|c|c|c|}
\hline $\begin{array}{l}\text { Universal and equitable access } \\
\text { to safe and affordable drinking } \\
\text { water for all (6.1) }\end{array}$ & +3 , Indivisible from child health & $\begin{array}{l}\text { Health and } \\
\text { nutrition are } \\
\text { outcomes }\end{array}$ \\
\hline
\end{tabular}

Provide universal access to safely managed, affordable, and sustainable drinking water

Invest in education on the importance of safely managed water use and infrastructure in households, communities, schools, and health facilities

\begin{tabular}{ll}
\hline Ensure access to modern & Depending on which strategies are \\
energy for all (7.1) & chosen, the effect on child health can be \\
& positive or negative. For example: +1, \\
modern energy replacing traditional solid \\
biomass cook stoves improves children's \\
respiratory health through reducing indoor \\
air pollution and outdoor pollution; -2, for \\
many countries, abundant energy means \\
fossil, nuclear, etc. energy, and might thus \\
be harmful for child health
\end{tabular}

Adopt policies, especially fiscal, +2, Equality reinforces health. Universal Health is wage and social protection access to healthcare reinforces greater outcome policies, and progressively equality

achieve greater equality (10.4)

Access to safe, affordable, accessible, and sustainable transport, including public transport; road safety (11.2)
+1 , Road safety and safe transportation reinforces child health in many ways, including access to health and education facilities, prevention of road accidents
Health is

outcome

Reduce household and ambient air pollution through the increased use of clean energy fuels and technologies in the home (for cooking, heating, lighting)

\author{
Environmentally sound \\ +1 , These measures are needed to \\ Health is \\ management of chemicals and minimise the adverse effects of chemical outcome \\ all wastes, reduction of their \\ release to air, water, and soil
} (12.4)
Provide entitlements for parental leave and for childcare for working parents and promote incentives for flexible work arrangements for men and women

Health is Provide safe transportation to health, education, and work facilities, outcome including during emergencies

Improve road safety, including through mandatory wearing of seat-belts and cycle and motorcycle helmets

Improve regulation and compliance of drivers, including introduction of a graduated driving licence that restricts driving options for inexperienced drivers

Eliminate non-essential uses of lead (e.g. in paint) and mercury (e.g. in healthcare and artisanal mining) and ensure the safe recycling of lead- or mercury-containing waste 